\title{
Qualitative Analysis of Illicit Heroin via Visual Examination for Forensic Intelligence
}

\author{
Kar-Weng Chan ${ }^{1,2 *}$, Guan-Huat Tan ${ }^{1}$ and Richard CS Wong ${ }^{1}$ \\ ${ }^{1}$ Department of Chemistry, University of Malaya, 50603 Kuala Lumpur, Malaysia \\ ${ }^{2}$ Department of Chemistry, Ministry of Science, Technology and Innovation (MOSTI), 46661, Petaling Jaya, Malaysia
}

\begin{abstract}
The qualitative information derived from the physical characteristics associated with heroin seizures has never been given emphasis. This study seeks to make use of the qualitative data for forensic intelligence purposes. A photographic setup is introduced for preserving the original conditions of the samples. The technique was found to be simple and inexpensive; it is also useful for general heroin profiling. The textures of the heroin substances, wrapping styles observed from the drug packages and the sealing on the package were documented. Upon analyzing 311 heroin cases, it was found that most cases contained coarse structures of heroin which were packed in packages bearing one packer's seal with rectangular seal-marks. Possible interpretations for the observed variables are also given in this paper.
\end{abstract}

Keywords: Forensic science; Qualitative analysis; Illicit heroin; Receptacles

\section{Introduction}

Illicit heroin has been the chief drug of abuse in Malaysia for decades. From the forensic intelligence perspective, establishing sample relationships could be a useful way to estimate the size of the heroin network within the local context. Many heroin profiling programs emphasize the determination of adulterants, opium alkaloids and manufacturing by-products present in the seized samples [1-5]. In this respect, gas chromatography and high performance liquid chromatography are often the separation techniques of choice. By manipulating the chemical data through chemometrics, the relationships between the samples can be estimated. In most cases, the samples could be related to the origins if samples of known origins are used for cluster analysis [6,7]. Despite its practicality, the analytical cost for the aforementioned analyses can be very high. Besides, the relevant discussions regarding the various analytical techniques involved in heroin profiling have also been presented $[8,9]$.

It is also commonplace that the cluster analysts may utilize physical measurements for statistical evaluation. By far, no emphasis is placed on the qualitative aspects of the heroin seizures. As a result, this paper aims to present the possible qualitative information that can be extracted from the heroin samples. It also highlights the utility of this information for forensic intelligence as per understanding the modus operandi of the dealer directly related to the seized drug.

\section{Materials and Methods}

\section{Sample collection}

A total of 311 heroin cases were sampled from all the submissions made in 2010. The heroin samples were subjected to ten aspects for visual examination and physical measurements, out of which only four qualitative aspects (photography, the texture of the drug substance, the wrapping style of the package and the sealing on the package) are discussed in this paper. Other aspects have been reported in the literature [10].

\section{Photography}

A representative sample unit was chosen from each case for photography. Figure 1 shows the photographic setup [11] adapted for obtaining a close-up for each case in order to preserve its original appearance. A representative sample was spread out on a polystyrene platform in the centre and a ruler bearing four standard hues (CMYK whereby $\mathrm{C}=$ Cyan, $\mathrm{M}=$ Magenta, $\mathrm{Y}=$ Yellow, $\mathrm{K}=$ Black) was placed next to the sample. The ruler gave a general measurement for the sample whereas the standard hues provided relative color adjustment for interpreting the photographs especially when different illumination was used for the photographs taken at two different times. Besides, two study lamps wrapped with tracing papers were installed on opposite

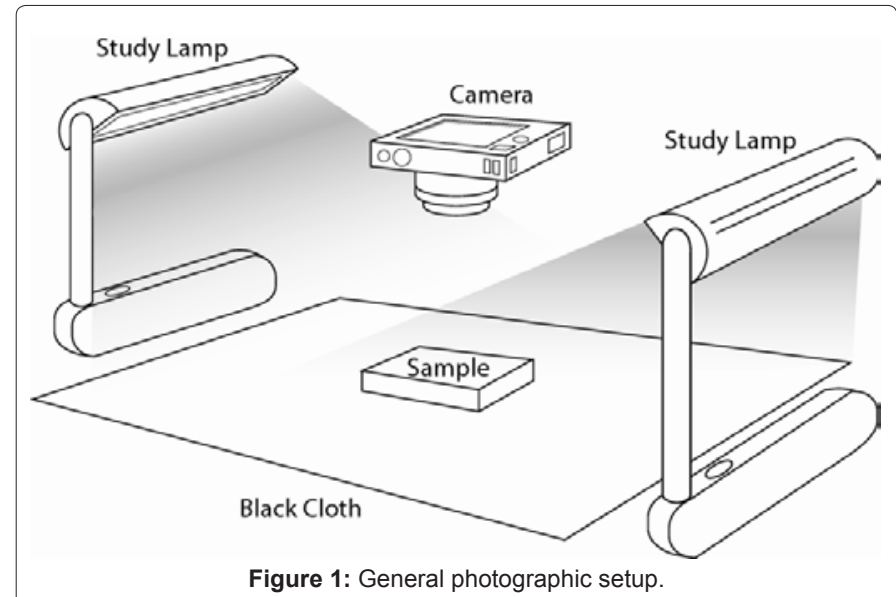

*Corresponding author: Kar-Weng Chan, Department of Chemistry, Ministry of Science, Technology and Innovation (MOSTI), Jalan Sultan, 46661 Petaling Jaya, Malaysia, Tel: 60123627609; Fax:60379581173; E-mail: chankarweng@ siswa.um.edu.my

Received June 09, 2012; Accepted June 19, 2012; Published June 21, 2012

Citation: Chan KW, Tan GH, Wong RCS (2012) Qualitative Analysis of Illicit Heroin via Visual Examination for Forensic Intelligence. J Forensic Res 3:159. doi:10.4172/2157-7145.1000159

Copyright: $\odot 2012$ Chan KW, et al. This is an open-access article distributed under the terms of the Creative Commons Attribution License, which permits unrestricted use, distribution, and reproduction in any medium, provided the original author and source are credited. 
sides to provide diffuse lighting to the sample. The lamps can be disregarded if there was sufficient lighting.

For the texture of the drug substance, three categories were selected to offer textural descriptions, and this was based solely on visual inspection with the following criteria (Table 1).

\section{Texture of heroin substance}

In most cases, some of the illicit heroin samples contained in clear packages were submitted together with additional wrappers such as newspapers and commercial plastic bags. To minimize irrelevant information, only the package/receptacle having direct contact with the substance was considered. To document the packages devoid of illicit manufacturer's markings, the plastic wrapping style can be generally defined using seal $(\mathrm{S})$, margin $(\mathrm{M})$ and portion $(\mathrm{P})$ as in Table 2.

With the predefined morphology, the wrapping of the plastic package was observed and recorded based on the following categories:

Type $1 \mathrm{a}$ - seals on brims, no margin, 1 portion

Type $1 \mathrm{~b}-2$ seals, 1 margin, 2 portions

Type 1c -3 seals, 1 margin, 2 portions

Type 2a - 2 seals, 2 margins, 3 portions

Type $2 \mathrm{~b}-3$ seals, 2 margins, 4 portions

Type $2 c-3$ seals, 3 margins, 6 portions

Type $3 \mathrm{a}-2$ seals, 2 margins, 5 portions

Type $3 b-3$ seals, 2 margins, 7 portions

Type $4 \mathrm{a}$ - tube with seal(s)

Tube $4 \mathrm{~b}$ - undefined

\section{Wrapping style}

Most of the plastic packets are usually accompanied by at least two seals. One seal at the bottom is normally made by the legitimate plastic manufacturer. Other seal(s) are most likely to have been introduced by the drug packer after the drug substances are placed into the plastic packets during packing. The number of such packer's seals together with the seal pattern and seal quality observed in all individual plastic packages were recorded based on 1) the number of packer's seals, 2) seal patterns that are defined according to Figure 2,3 clarity of the seal.

Number of packer's seals: Not applicable

Single

Double

Triple

\begin{tabular}{|c|c|}
\hline Category & Criteria \\
\hline Coarse & $\begin{array}{l}\text { - Apparently large and visually well-defined bodies of } \\
\text { substance } \\
\text { - A small amount or less than half of the sample is in the } \\
\text { form of a fine powder }\end{array}$ \\
\hline Coarse and Fine & $\begin{array}{l}\text { - Both visually defined bodies of substance and fine } \\
\text { powder are present in approximately equal portions }\end{array}$ \\
\hline Fine & $\begin{array}{l}\text { - Powdery appearance } \\
\text { - A small amount or less than half of the sample is in the } \\
\text { form of a coarse substance }\end{array}$ \\
\hline
\end{tabular}

Table 1: Texture categories for the description of heroin substance.

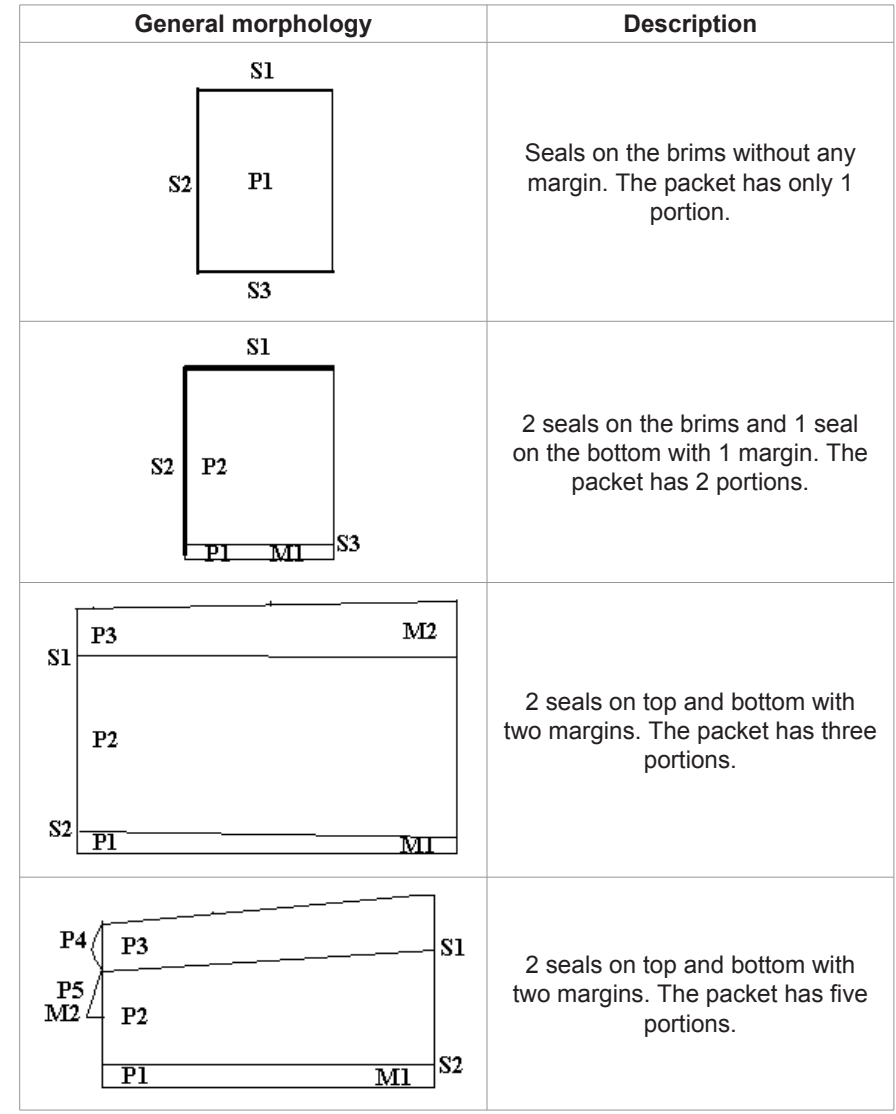

Table 2: General morphology of plastic wrapping styles defined by seal, margin and portion.

\section{Seal pattern/type: No seal \\ Undefined seal pattern}

Rectangle

Clip seal

Seal quality (clarity):Not applicable

Clear and complete

Clear but incomplete

Vague but complete

Vague and incomplete

\section{Results and Discussion \\ Photography}

To date, there has not been any published work detailing the procedure of photography for packaged heroin. The photographic setup adopted in this study was inexpensive and user-friendly. Through photography, all crucial details such as the geometrical shape of the plastic package, the coarseness of the heroin substance and many other qualitative data can be clearly recorded in a color photograph.

Without the use of expensive instruments, two prevailing types of errors associated with the photographic setup are notable; namely 1) the error inherently associated with the substance and 2) the error arising from the setup and environment. The first type of error is common in powdery samples which always exhibit inconsistencies of 
color and particle size. The irregular particle surfaces tend to give rise to visual confusion as the lights are reflected in random directions from the particles and thus displaying varying tones in its color. In addition, the error is highly dependent on the positions of the particles and light reflections. Besides, reflections from the plastic surface and colored markings were another potential source of error which could not be easily eliminated during photography. The second type of error is the variation associated with the lighting as well as the angle at which the camera was held.

In view of these two errors, the photographs obtained were therefore neither repeatable nor reproducible. However, with a CMYK calibrator placed next to the sample, the second type of error can be generally addressed. The photographs shown in Figure 3 clearly depict the possible visual distortion imparted by the irregular surfaces and the lighting for a sample photographed at three different times in a series. Despite the poor precision between the photographs, the strips of the CMYK calibrator in the photographs provide a reference to show how much the color deviates in each series. It is observed that all the series show varying degrees of brightness or darkness. However, this factor does not significantly affect the color of the heroin substance. To the minimum extent, the substance colors in these three individual cases are readily decipherable and distinguishable.

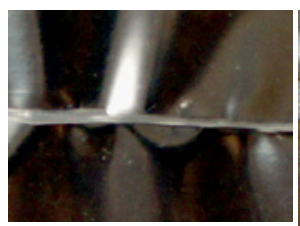

(a)

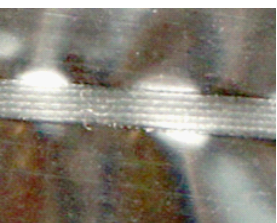

(b)

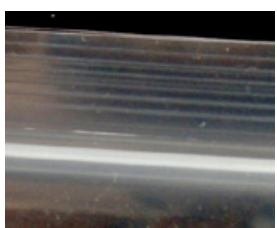

(c)
Figure 2: Appearance of different seal patterns (Three major patterns are (a) undefined seal pattern; (b) rectangle; (c) clip seal).

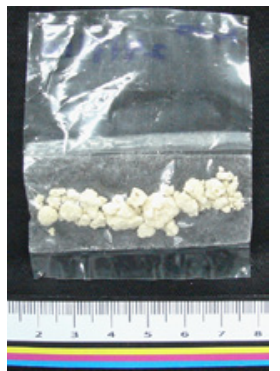

293-a

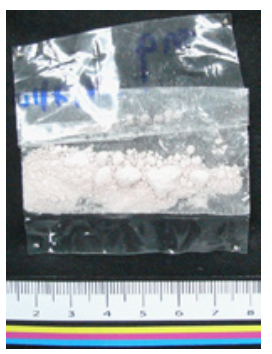

294-a

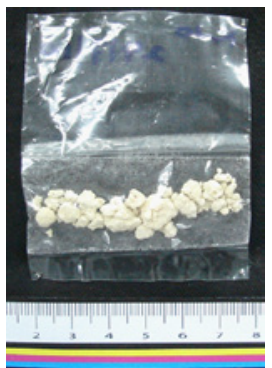

293-b

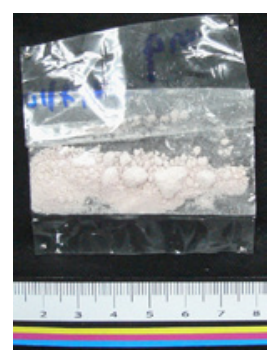

294-b

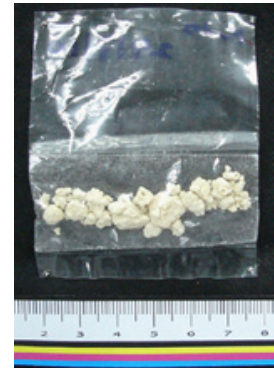

$293-\mathrm{c}$

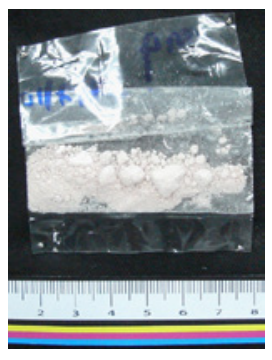

294-c
Figure 3: A comparison of three different original photographs of three samples taken at different times without photo editing.
Coarse \& Fine, $11.3 \%$

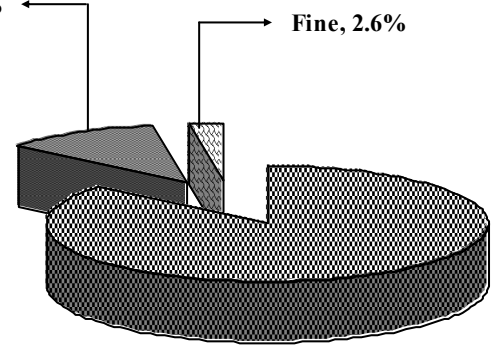

Coarse, $86.2 \%$

Figure 4: Distribution of substance texture in 311 cases taken for profiling

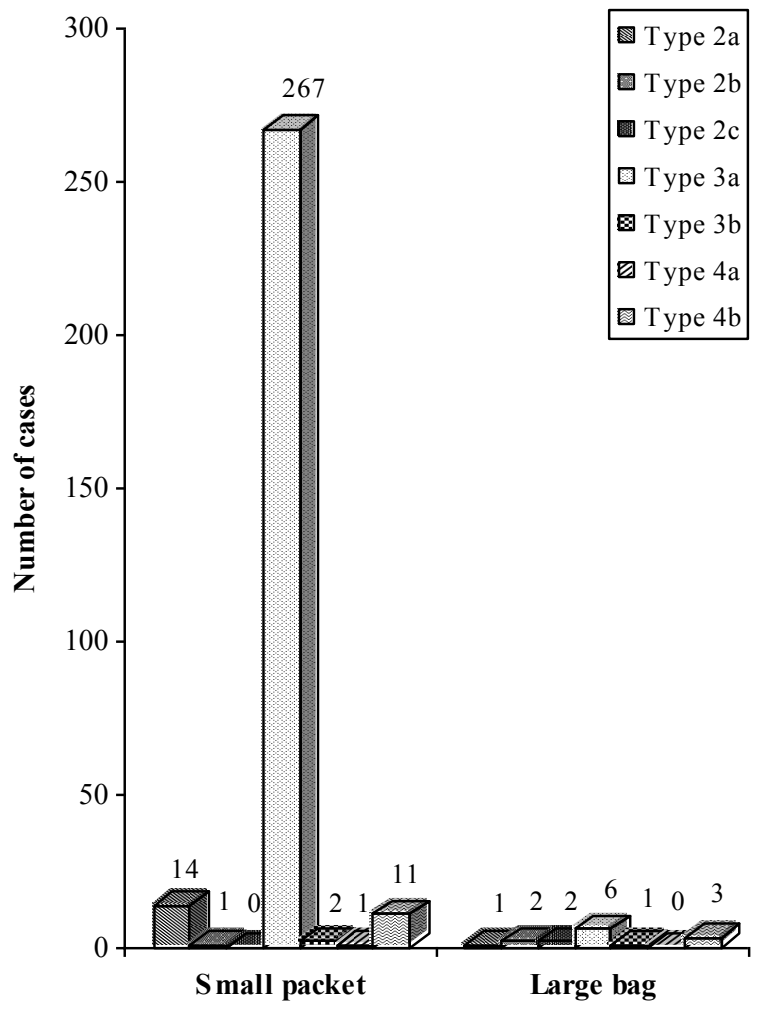

Figure 5: Frequency of wrapping styles in 311 cases taken for profiling.

\section{Texture of heroin substance}

In this study, almost all samples contained both fine powders and coarse granules. However, it is inaccurate to characterize the intended condition of these samples as 'coarse and fine' structures, since handling and shipment can also give rise to the dislodgement of the fine powders and particles from the main coarse structures. In this regard, small amounts of fine powders in the sample were disregarded when coding the texture as the 'coarse' structure. However, if there is a significant amount of the fine powders (approximately $50 \%$ of the sample) together with an equal amount of the coarse structures present in the majority of the sample units of a single case, then it was justified to group them in the 'coarse and fine' category. Hypothetically, the presence of very large quantities of fine powders (in the 'fine' category) is often the result of grinding and crushing during packing. In contrast, rough handling would usually not produce more than $50 \%$ fine 
powders in the majority of the sample units. Guided by the foregoing rationales, $86.2 \%, 11.3 \%$ and $2.6 \%$ of the 311 cases were respectively categorized into 'coarse', 'coarse and fine', and 'fine' categories (Figure 4). In particular, the 'coarse' category plays an important role in distribution. The coarseness of the illicit heroin under this study varied from approximately $3 \mathrm{~cm}$ to a fraction of $1 \mathrm{~mm}$. Coarse substances are space consuming and require tedious handling procedures as compared to the fine powder. However, its $86.2 \%$ prevalence signifies the importance of this structure in the distribution process. If the illicit heroin is kept as powders, it will increase the surface exposure of heroin/diamorphine to atmospheric moisture and thus speeding up undesirable decomposition of diamorphine to 6-monoacetylmorphine. So, the mass of heroin was not usually crushed into powders. Instead, large granules were maintained as such in the distribution so as to reduce the surface area. Limited surfaces for water interaction will therefore reduce the effects of deacetylation.

\section{Wrapping style}

The wrapping style could be used as a screening criterion to determine if the sample units were from the same batch (referring to the batch at the packing level). In general, if two samples appear as yellow substances but with two different wrapping styles, they are treated as two individual cases. In line with the concept of modus operandi, the chosen wrapping style is the common mode of operation adopted by the packer/syndicate. Therefore substances originating from the same batch could have been packed in a similar style. The wrapping styles encountered in the 311 heroin cases comprised seven major types and the frequency is presented in Figure 5. However, Types $1 \mathrm{a}, 1 \mathrm{~b}$ and $1 \mathrm{c}$ were not encountered in the cases. Among all, Type $3 \mathrm{a}$ ( 2 seals, 2 margins and 5 portions) was the most commonly employed wrapping style in Malaysia. In addition, folding the packet may also help to shorten the extra length of the plastic packet. For shorter plastic packets, Type $2 \mathrm{a}$ (without a fold) instead, was occasionally employed.
The choice of other less common styles would also signify that there could have been other dealers whose common mode of packing was not associated with Type $3 \mathrm{a}$. These minor styles could be the signature wrapping styles adopted by these dealers.

\section{Sealing}

The sealing characteristics may indicate the mode of packing. All packages were sealed and Table 3 indicates that $94.9 \%$ of the cases originated from one particular sealing procedure. A single seal (referring to that deliberately made by the packer) is common because it is the most rapid way to seal off the opening of the receptacle for the purpose of securing the substance. Packages with two and three seals were rare, accounting for 14 and 2 cases respectively. Additional sealing was usually made on other edges of a plastic packet/bag which were cut open. Besides, $83.0 \%$ of the cases had seals bearing rectangular patterns whereas $16.7 \%$ had undefined patterns. The rectangular pattern is the impression transferred from the sealing base to the plastic film when the hot sealer's blade is pressed against it. Usually, a new sealing machine will show clear patterns on the plastic film. For undefined patterns, it could be due to two reasons. First, the machine is old and due to prolonged usage, the impression is no longer prominently displayed on the plastic film. Second, the sealer's blade is excessively hot until it melts or distorts the impression.

According to Table 3, it is also recorded that 203 cases showed clear and complete seals, compared to 92 cases with vague and complete seals. The latter occurrence was probably due to poor pressing and insufficient heating during sealing.

\section{Conclusion}

The present study demonstrated that the qualitative information obtained from the heroin samples may be useful to provide intelligence information. It offers indications for the mode of operation adopted by the heroin dealer. More importantly, this study seeks to verify that

\begin{tabular}{|c|c|c|c|c|c|c|c|c|c|c|}
\hline \multirow{3}{*}{ No. of seals } & \multirow{3}{*}{ Seal clarity } & \multicolumn{8}{|c|}{ Seal type } & \multirow{3}{*}{ Tota } \\
\hline & & \multicolumn{2}{|c|}{ Not Applicable } & \multicolumn{2}{|c|}{ Undefined } & \multicolumn{2}{|c|}{ Rectangle } & \multicolumn{2}{|c|}{ Clip } & \\
\hline & & $\mathrm{s}$ & $\mathbf{L}$ & s & $\mathbf{L}$ & $\mathbf{S}$ & $\mathbf{L}$ & $\mathbf{S}$ & $\mathbf{L}$ & \\
\hline 0 & - & 0 & 0 & - & - & - & - & - & - & 0 \\
\hline \multirow{4}{*}{1} & $C \& C$ & - & - & 13 & 0 & 184 & 5 & 1 & 0 & 203 \\
\hline & C \& inC & - & - & 0 & 0 & 0 & 0 & - & - & 0 \\
\hline & $V \& C$ & - & - & 36 & 1 & 54 & 1 & - & - & 92 \\
\hline & $V$ \& inC & - & - & 0 & 0 & 0 & 0 & - & - & 0 \\
\hline \multirow{4}{*}{2} & $C \& C$ & - & - & 0 & 0 & 7 & 6 & - & - & 13 \\
\hline & C \& inC & - & - & 0 & 0 & 0 & 0 & - & - & 0 \\
\hline & $V \& C$ & - & - & 0 & 1 & 0 & 0 & - & - & 1 \\
\hline & V \& inC & - & - & 0 & 0 & 0 & 0 & - & - & 0 \\
\hline \multirow{4}{*}{3} & $C \& C$ & - & - & 0 & 0 & 0 & 1 & - & - & 1 \\
\hline & C \& inC & - & - & 0 & 0 & 0 & 0 & - & - & 0 \\
\hline & $V \& C$ & - & - & 1 & 0 & 0 & 0 & - & - & 1 \\
\hline & $V$ \& inC & - & - & 0 & 0 & 0 & 0 & - & - & 0 \\
\hline \multicolumn{2}{|c|}{ Total } & 0 & 0 & 50 & 2 & 245 & 13 & 1 & 0 & 311 \\
\hline
\end{tabular}

$\mathrm{S}=$ Small packet; $\mathrm{L}=$ Large packet

C \& C = Clear and complete; C \& inC = Clear but incomplete

V \& $C=$ Vague but complete; $V \&$ in $C=$ Vague and incomplete

Table 3: Frequency of seal patterns and seal clarity according to the number of seals. 
Citation: Chan KW, Tan GH, Wong RCS (2012) Qualitative Analysis of Illicit Heroin via Visual Examination for Forensic Intelligence. J Forensic Res 3:159. doi:10.4172/2157-7145.1000159

Page 5 of 5

every single piece of information is a forensic clue for investigation. Therefore, all forensic practitioners should not solely rely on the chemical data since the physical aspects (especially those of the qualitative nature) are also crucial to provide a complete story for the case.

\section{Acknowledgement}

We would like to thank the Department of Chemistry Malaysia for providing the facilities to carry out this project.

\section{References}

1. Zhang D, Shi X, Yuan Z, Ju H (2004) Component analysis of illicit heroin samples with GC/MS and its application in source identification. J Forensic Sci 49: 81-86.

2. Zelkowics A, Magora A, Ravreby MD, Levy R (2005) Analysis of a simulated heroin distribution chain by HPLC. J Forensic Sci 50: 849-852.

3. United Nations Office of Drugs and Crime (2005) Methods for impurity profiling of heroin and cocaine. Vienna: United Nations.

4. Strömberg L, Lundberg L, Neumann H, Bobon B, Huizer H, et al. (2000)
Heroin impurity profiling: A harmonization study for retrospective comparisons. Forensic Sci Int 114: 67-88.

5. Morello DR, Cooper SD, Panicker S, Casale JF (2010) Signature profiling and classification of illicit heroin by GC-MS analysis of acidic and neutra manufacturing impurities. J Forensic Sci 55: 42-49.

6. Myors RB, Crisp PT, Skopec SV, Wells RJ (2001) Investigation of heroin profiling using trace organic impurities. Analyst 126: 679-689.

7. Myors R, Wells RJ, Skopec SV, lavetz R, Skopec P, et al. (1998) Preliminary investigation of heroin fingerprinting using traces element concentrations. Anal Commun 35: 403-410.

8. Besacier F, Chaudron-Thozet H (1999) Chemical profiling of illicit heroin samples. Forensic Sci Rev 11: 105-119.

9. Dams R, Benijts T, Lambert WE, Massart DL, De Leenheer AP (2001) Heroin impurity profiling: Trends throughout a decade of experimenting. Forensic Sci Int 123: 81-88.

10. Chan KW, Tan GH, Wong RCS (2012) Investigation of illicit heroin seized in Malaysia: Physical characteristics and chemical profiling. Aus. J Forensic Sci

11. Freeman M (2004) Close-up photography. Cambridge, England ILEX. 